This item was submitted to Loughborough's Research Repository by the author.

Items in Figshare are protected by copyright, with all rights reserved, unless otherwise indicated.

Hollow golf club head modal characteristics: determination and impact applications

PLEASE CITE THE PUBLISHED VERSION

http://dx.doi.org/10.1007/BF02321657

PUBLISHER

Society for Experimental Mechanics (SEM) @ Springer

VERSION

AM (Accepted Manuscript)

LICENCE

CC BY-NC-ND 4.0

REPOSITORY RECORD

Hocknell, Alan, Sean R. Mitchell, Roy Jones, and Steve Rothberg. 2019. "Hollow Golf Club Head Modal Characteristics: Determination and Impact Applications". figshare. https://hdl.handle.net/2134/9660. 
This item was submitted to Loughborough's Institutional Repository (https://dspace.lboro.ac.uk/) by the author and is made available under the following Creative Commons Licence conditions.

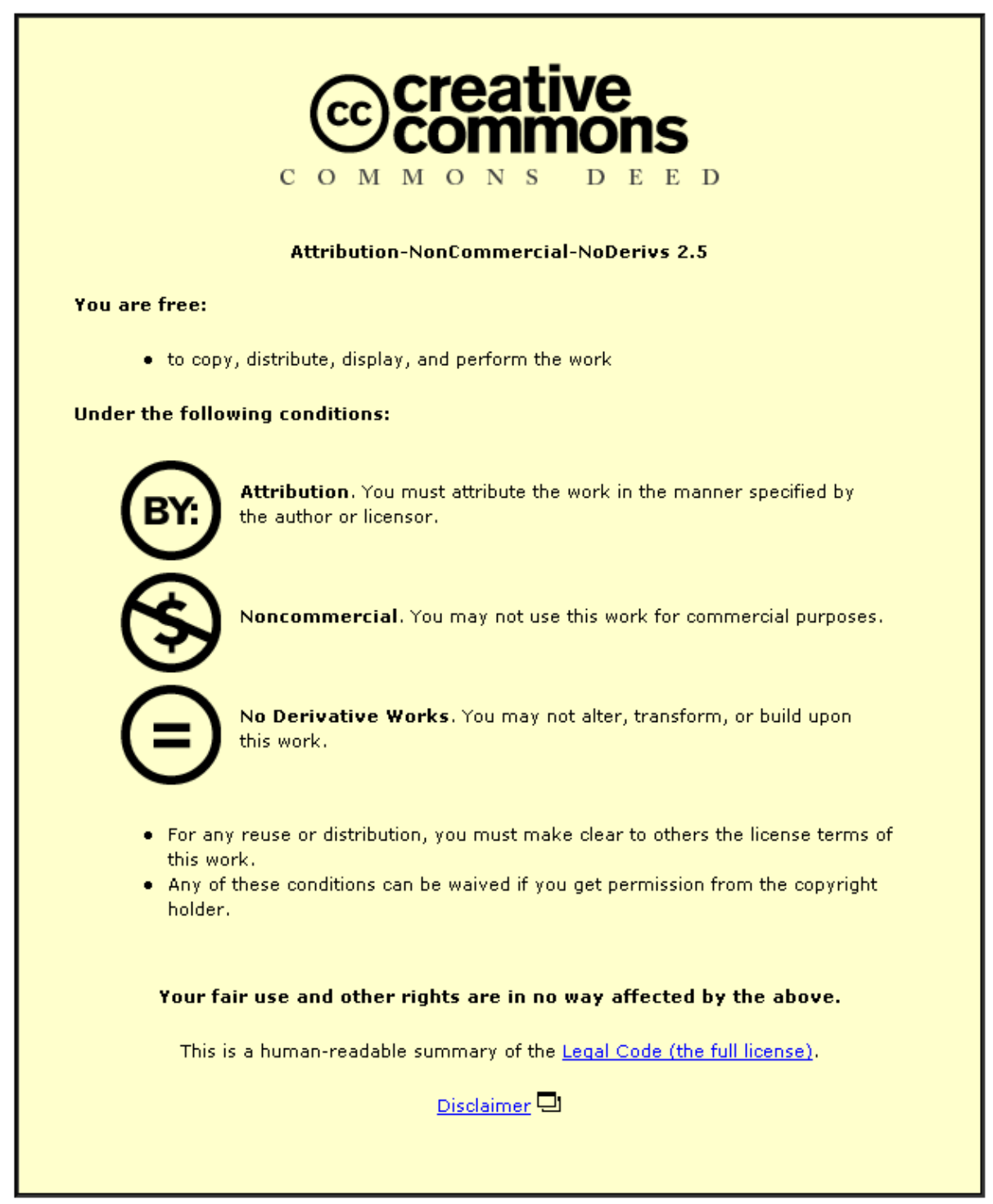

For the full text of this licence, please go to: http://creativecommons.org/licenses/by-nc-nd/2.5/ 


\title{
Hollow golf club head modal characteristics : Determination and impact applications
}

\section{A Hocknell, SR Mitchell, R Jones and SJ Rothberg \\ Loughborough University}

\section{Leicestershire, LE11 3TU}

\section{UK}

\begin{abstract}
The design of modern hollow golf club heads is a labour intensive process involving extensive performance testing both by robotic and real golfers. This paper describes how, by correlating club head mechanical behaviour with functional performance, it will become possible to use validated computational models to predict this performance as well as related contributions to the ill-defined concept of 'feel'. Successful use of experimental modal analysis to validate a hollow golf club head finite element model is reported. Modal tests employing non-contacting laser based transducers facilitated identification of the natural frequencies and corresponding modeshapes for the three main surfaces of the club head. The experimental data suggests predominantly different modal characteristics for each surface and this compares favourably with equivalent data obtained from the finite element model. The modal data is also used to identify surfaces responsible for particular frequency components present in the club head impact sound spectrum. The potential for detailed impact performance prediction using the finite element model is further demonstrated by comparison of computed and experimental club head acceleration measurements recorded during simulated and actual club-ball impacts.
\end{abstract}




\section{Introduction}

Golf club design and manufacture has become increasingly complex in response to player demands for improvements in equipment performance. The design to manufacture lead time can be considerable when developing new products since the industry is still conditioned by traditional values and practices. A craftsman generally sculpts an aesthetically pleasing shape with those features thought to enhance performance and the prototypes are play tested using robots and humans until a satisfactory design is obtained. This process, coupled with increasing performance demands has lead to long and expensive new product introduction (NPI) timescales. New approaches to design and prototype realisation are therefore necessary.

Computer aided design (CAD) can reduce the time required to generate club head models and can also provide exact specification of shape for the production of prototypes by computer numerical control machining. Design analysis using finite element methods can provide relevant force, stress and displacement data for a simulated golf shot. However, relatively few finite element impact studies have been reported [1-5] and in every case it appears that models have been limited by inaccuracies in modelling the sculptured shape or difficulties in obtaining experimental data to validate the model behaviour during impact. The use of modal analysis has been previously directed at modes of vibration of the club which are dominated by the shaft and where the club head is treated as a rigid body [6-10]. The period of oscillation of these modes is typically in the range $1.5-50 \mathrm{~ms}$ which is significantly longer than the impact duration of around $450 \mu \mathrm{s}$. Thus, the effect of the shaft dominated modes during 
impact is of less significance than the effect of the higher frequency club head modes in the study of club head performance during impact.

The requirement for model validation during the high speed impact of lightweight bodies has driven the development of a measurement system employing laser based non-contacting methods to determine accurate point velocity measurements throughout impact [11]. In what follows this research is extended to the modal analysis of hollow club heads by using non-contacting laser based measurements to determine the natural frequencies of vibration and corresponding modeshapes of a hollow stainless steel "wood". The information thus gained has been used to verify the accuracy of the finite element model of the club head in the frequency range relevant to golf club-ball impact. The reliability of the finite element model has been further proven by favourable comparison with club head vibration data measured during impact with a golf ball and also with the higher frequencies present in the sound of impact between a hollow club head and a golf ball, an important aspect of 'feel' in the golf shot [12]. Models of this type can be used to predict mechanical behaviour which can be related to equipment performance.

\section{Representation of the structure}

\subsection{Club head geometry model}

Geometrical accuracy is essential if the model is to be used in a predictive capacity but accurate models of sculptured surface structures are generally more difficult to produce than prismatic structures. In this study an existing club head shape was 
reverse engineered into a $\mathrm{CAD}$ system by digitising the external shape features and cutting the head to obtain internal features and part thicknesses. A 3-D co-ordinate measuring machine with a touch trigger probing system was used to measure the surface and this data was used to create a model on the DUCT surface modelling software. The creation of an accurate geometry model in such software is the start point of the advanced design and analysis procedure advocated in this paper. The club head was modelled using the extended form feature methodology $[13,14]$, where the primary features of the club are modelled far beyond their actual boundaries and blended with secondary features, the excess being trimmed away. The selection of primary features is of considerable importance for ease of modelling and design modification. In this case the club head was considered to consist of five primary features, namely the face, crown, sole, bowl and hosel. A feature anatomy for the club is shown in figure 1. All of the primary form features were digitised and created as extended forms in DUCT. However, the blends were created approximately by using the rolling ball filleting facilities of the software. A wire frame model of the club head is shown in figure 2 with primary form features labelled. The surface is modelled to an accuracy of better than $0.14 \mathrm{~mm}$ and the section thicknesses were measured to an accuracy of $0.01 \mathrm{~mm}$ using a pointed anvil micrometer. Areas of the model where there has been preferential thickening, e.g. under logos, have not been modelled and may be a source of slight inaccuracy.

\subsection{Finite element mesh generation}

The discretisation of the geometry model into a mesh of finite elements well suited to the physical situation under investigation is crucial to the quality of the analysis 
solution. The sculptured nature of the golf club head makes mesh generation difficult if the entire club head is considered as a single surface because elements are produced with excessive warp and taper or with aspect ratios unsuitable for finite element analysis. A feature based approach to quadrilateral mesh generation on sculptured surface structures has been previously reported [15]. From a structural viewpoint, primary features are always of importance. In the hollow golf club head anatomy, the secondary features are involved in areas of the product's surface geometry which have stress raising characteristics and are thus retained in the discretised model. Tertiary features, such as manufacturers' logos, are rarely included in structural analysis as the difficulty of discretising these often small and complex markings significantly outweighs the structural importance of the feature.

The finite element mesh created using the procedure reported in [15] is shown in figure 3. This mesh was constructed within the MSC/PATRAN pre/post processing environment and contains 701 2-dimensional quadrilateral shell elements with thicknesses determined from pointed anvil micrometer measurements on the real club head. The thicknesses range from $0.6 \mathrm{~mm}$ in areas of the bowl feature to $3.5 \mathrm{~mm}$ at the bottom of the face. The Young's Modulus of the material was determined by static tensile tests on a specimen cut from a stainless steel investment cast club head and was found to be $207 \mathrm{GN} / \mathrm{m}^{2}$. The density of the material is $\rho=7850 \mathrm{~kg} / \mathrm{m}^{3}$, which when applied to the finite element model gives a club head model a mass of 190 grammes. This is 3grammes less than the mass of the real clubhead due to the exclusion of a small internal shaft support from the discretised model. 


\section{Computational and experimental modal analysis}

\subsection{Modal analysis of the finite element model}

It is generally accepted that corroboration of the first few modes of vibration with experimental data obtained in a modal test of the same structure can provide reassurance of the basic validity of the model [16]. In this study the hollow golf club head model was constrained at the top of the hosel in all directions and natural frequencies and modeshapes of the model were calculated in the frequency range 0$20 \mathrm{kHz}$.

\subsection{Experimental modal testing of the hollow golf club head}

A harmonic vibration excitation was applied to the real hollow golf club head by a small piezo-ceramic tile bonded to the sole plate. The club head was held rigidly at the top of the hosel to replicate the boundary conditions in the finite element model and a broadband sinusoidal excitation was applied to the piezo-ceramic tile over the frequency range $0-20 \mathrm{kHz}$. The low mass of each primary feature of the hollow golf club head dictated that it was appropriate to make pointwise frequency response measurements using a non-contacting laser Doppler vibrometer (LDV) instead of a more traditional piezo-electric accelerometer. The vibrometer focuses a laser beam down to a small spot on the target, collecting the returning light in direct backscatter. According to the Doppler principle, the frequency of the light returning from the target is shifted in proportion to the component of the target velocity which lies along the axis of the incident laser beam. By electronically tracking this frequency shift, the vibrometer can measure this component of the target velocity. Thus the ratio of the 
target surface velocity to the piezo-ceramic tile input voltage was used to determine the frequency response function for several measurement positions on the face, crown and sole features.

In order to obtain the corresponding vibration modeshape for each natural frequency of the hollow club head, the club head was excited by a single frequency sinewave at each of the natural frequencies identified using the vibrometer. The modeshapes could have been constructed from an array of pointwise LDV measurements, but it is more convenient to observe the modeshapes using Electronic Speckle Pattern Interferometry (ESPI) [17]. This wholefield laser technique involves using an expanded laser beam to cover the target. The light incident on a moving target undergoes a phase change which can be used to determine target displacement information. The output from the system is in the form of a time averaged image of the target showing light and dark fringes. Each fringe is a contour of constant vibration displacement amplitude, hence antinode and node regions can be identified at each natural frequency.

\subsection{Comparison of results}

The natural frequencies of vibration were calculated using the finite element model and measured experimentally using the LDV for the face, crown and sole of the hollow golf club head. These three primary features together comprise the majority of the hollow shell structure. The lowest mode of vibration of a primary surface feature is $3.62 \mathrm{kHz}$. Modes of vibration of a golf club below this frequency are dominated by vibration of the shaft and frequencies present in the impact sound below $3.62 \mathrm{kHz}$ have been previously shown to be dominated by vibration of the ball [12]. Club head 
vibrations at frequencies above $20 \mathrm{kHz}$ have not been observed during extensive impact experiments in which measurements from a piezo-electric accelerometer were used to analyse club head vibration during impact [11]. Hence the frequency range of interest to the analysis of golf club heads is $3.6-20 \mathrm{kHz}$ and table 1 shows good agreement between computational and experimental results for modes of vibration in this frequency range.

\begin{tabular}{cccccc} 
& Active Surface & \multicolumn{4}{c}{ Natural Frequency (kHz) } \\
Face & Crown & Sole & FE Model & Experiment & \% Difference \\
& $\checkmark$ & $\checkmark$ & 3.72 & 3.62 & 2.76 \\
& $\checkmark$ & $\checkmark$ & 4.95 & 5.05 & -1.98 \\
$\checkmark$ & & & 5.51 & 5.64 & -2.30 \\
$\checkmark$ & $\checkmark$ & & 6.09 & 5.96 & 2.18 \\
$\checkmark$ & $\checkmark$ & $\checkmark$ & 6.48 & 6.40 & 1.25 \\
$\checkmark$ & $\checkmark$ & & 6.68 & 6.77 & -1.33 \\
$\checkmark$ & $\checkmark$ & & 7.20 & 7.40 & -2.70 \\
$\checkmark$ & & & 7.84 & 7.70 & 1.82 \\
& $\checkmark$ & $\checkmark$ & 8.15 & 8.20 & -0.61 \\
$\checkmark$ & $\checkmark$ & $\checkmark$ & 8.88 & 8.81 & 0.79 \\
$\checkmark$ & $\checkmark$ & $\checkmark$ & 10.40 & 10.11 & 2.87 \\
& $\checkmark$ & $\checkmark$ & 10.80 & 10.71 & 0.84 \\
$\checkmark$ & $\checkmark$ & $\checkmark$ & 11.90 & 11.84 & 0.51 \\
$\checkmark$ & & & 12.84 & 12.78 & 0.47 \\
$\checkmark$ & $\checkmark$ & $\checkmark$ & 13.40 & 13.30 & 0.75 \\
& $\checkmark$ & & 13.87 & 13.95 & -0.57 \\
$\checkmark$ & & & 14.45 & 14.40 & 0.35 \\
$\checkmark$ & $\checkmark$ & & 15.10 & 15.03 & 0.47 \\
$\checkmark$ & $\checkmark$ & $\checkmark$ & 16.20 & 16.38 & -1.10 \\
$\checkmark$ & $\checkmark$ & $\checkmark$ & 18.23 & 18.30 & -0.38 \\
& $\checkmark$ & $\checkmark$ & 19.75 & 19.70 & 0.25
\end{tabular}

Table 1 - Computed and experimentally measured natural frequencies of vibration of the hollow club head face, crown and sole.

The data in the table suggests that some modes of vibration are specific to a single surface whilst others are common to more than one surface. This is confirmed in the frequency response functions of the face, crown and sole plotted in figure 4 , where 
some peaks are common to more than one frequency response function. The significance of this effect is discussed in section 4 .

The computational and experimental results for the crown show slightly greater disparity than the face and sole results. This is attributed to a surface digitisation error which occurred on the crown and created an unwanted ripple in the modelled surface. The ripple was removed by averaging over the surface, but this left an average geometrical error over the crown surface model of $0.14 \mathrm{~mm}$, which is significantly greater than the corresponding error in the face and sole geometries. This indicates the level of modelling accuracy required to achieve good agreement between the model and experimental data. The experimentally obtained natural frequencies have a measurement tolerance of $\pm 1 \%$.

The level of agreement shown in the natural frequency data presented in table 1 provides evidence for the basic validity of the finite element model. Further confirmation of the model validity is obtained by comparison of the corresponding modeshapes calculated on the finite element model and measured using the ESPI technique. Figure 5a shows the ESPI image for the first mode of vibration of the face. An antinode can be seen in the centre of the face, whilst the region around the edge of the face is a node. The equivalent mode calculated by the finite element model is shown in figure $5 \mathrm{~b}$ and displays the same modeshape. Example crown and sole modes of increasing complexity are shown in figures 6 and 7. Figure 6 shows the experimental observation and finite element prediction of the crown modeshape at $5.96 \mathrm{kHz}$ and 
figure 7 shows equivalent data for the sole modeshape at $8.20 \mathrm{kHz}$. These results are considered to confirm the basic reliability of the finite element model.

\section{Discussion}

The geometrical accuracy of the CAD model and the discretisation of this geometry into a finite element mesh are of paramount importance in obtaining a finite element model which accurately predicts behaviour of the real club head. However, small errors in the $\mathrm{CAD}$ representation due to measurement and modelling inaccuracies are difficult to eliminate. Further inaccuracy is introduced by choosing not to include features such as internal weld beads and a small internal shaft support in the discretised model. The close agreement between the computational and experimental results presented in this paper has, however, shown the absence of these features from the model to have only a marginal effect. Having proven the validity of the hollow club head modelling process, the finite element model can then be used in a predictive capacity.

Immediate benefit can be gained from performing an inertia properties calculation based upon the distribution of element mass. This type of calculation can be performed quickly by finite element software and provides estimates of the centroid location and the principal moments of inertia. These mechanical attributes are difficult to determine experimentally for sculptured shapes and are important in golf club head design.

Analysis of club head vibrations which are excited during impact with a golf ball is important to the improved understanding of the energy transfer mechanisms between 
club head and ball and is also relevant to prediction of the impact sound. To the author's knowledge, there has been very little study of the high frequency energy transfer between a hollow club head and a golf ball. The natural frequency and mode shape results presented in this paper suggest significant modal activity in the impacting region of the club face at frequencies relevant to the impact duration. This warranted the further computational and experimental investigation conducted.

Introduction of a finite element golf ball model to the existing club head model was the only reliable way to reproduce the spatial and temporal loading variations which occur during impact, requiring the use of significant computing power in the analysis of this non-linear transient event. This analysis can, however, provide detailed insight into the mechanical behaviour of a golf club head during impact. Previous work [11] demonstrated how club head vibration during impact is related to the pattern of deformation and recovery of the golf ball. Figure 8a shows the acceleration history measured at the back of the club head by a piezo-electric accelerometer during impact with a golf ball. The equivalent finite element club/ball impact calculation is shown in figure $8 \mathrm{~b}$. Despite the complexity of the motion, agreement is clearly apparent and demonstrates the potential to use the model in further study of energy transfer during impact. This work may consider the frequency spectrum of vibrations excited in the club face during impact, its relation to the natural frequencies and modeshapes and the correlation with functional performance.

The sound heard by the golfer following impact with a golf ball is generated as a result of both club head and ball surface vibrations set up during the impact and which persist 
for approximately $2 \mathrm{~ms}$. Previous investigation [12] has shown that sound is an important component of 'feel' in the golf shot. The lower frequency components of the impact sound were shown to be due to vibration of the ball, whilst the higher frequency components are due to the hollow metal club head vibrating at its natural frequencies. Hence the modal data presented in this paper may be employed in the analysis of impact sounds.

The sound spectrum measured during the impact between a golf club head and a golf ball is shown in figure 9 for the frequency range $4.5-7.5 \mathrm{kHz}$. This data, which pertains to an impact involving the same club head as modelled in section 2 , is slightly more variable than the frequency response data shown in figure 4 due to a Doppler shift of around $100 \mathrm{~Hz}$ caused by movement of the club head away from the microphone during the measurement period. Despite the slight inaccuracy, several distinct peaks are visible in the spectrum indicating that the majority of the sound energy produced by vibration of the hollow club head surfaces is contained in several distinct frequencies. By comparison with the data in table 1 , it can be seen that a number of the peaks correspond to natural frequencies of vibration of the face, crown and sole. These are indicated by the letters ' $\mathrm{F}$ ', ' $\mathrm{C}$ ' and ' $\mathrm{S}$ ' above the corresponding peaks on figure 9. More significantly, the largest peaks occur at around $5.9 \mathrm{kHz}, 6.4 \mathrm{kHz}$ and $6.8 \mathrm{kHz}$ which are, by reference to table 1 , frequencies at which the vibration modeshape involves more than one surface.

The modeshape data obtained in this study indicates that the modes of vibration of the face in the frequency range $5-7 \mathrm{kHz}$ have a single antinode. The location of this 
antinode varies in a region around the face centre. The $5.9 \mathrm{kHz}$ and $6.8 \mathrm{kHz}$ face modeshapes exhibit an antinode approximately in the face centre whilst that of the $6.4 \mathrm{kHz}$ mode is to the left of centre. Figure 10 shows the $5.9 \mathrm{kHz}$ and $6.4 \mathrm{kHz}$ modeshapes calculated using the finite element model. Thus, the relative excitation of the face modes will be different for impacts between the club head and a ball occurring in the face centre and those which are off-centre. This is confirmed in the sound spectrum measured during an impact in which the contact was $15 \mathrm{~mm}$ to the left of the face centre, as shown in figure 11. By comparison with figure 9 , it can be seen that the contribution of frequency components in the range $5-6 \mathrm{kHz}$ is significantly reduced whilst the $6.4 \mathrm{kHz}$ component is increased. Hence the overall sound of the off-centre hit is of higher frequency and this is confirmed by subjective data gathered from a group of low handicap and professional golfers in [12]. Using the finite element modal analysis data, a club head designer could have predicted the main feature of the experimental results shown in figures 9 and 11 with some confidence at an early stage in the design process. This provides a basis for further research on the impact sound characteristics preferred by golfers and the ability to achieve these 'feel' characteristics by re-distributing the mass and stiffness of individual surfaces whilst simultaneously satisfying the inertia property requirements of the whole club head..

\section{Conclusion}

This paper describes a modelling and analysis capability for hollow golf club heads of significantly greater accuracy and superior detail to that previously reported. This capability is important to the development of greater understanding of club head mechanical behaviour during impact in terms of energy transfer and 'feel' 
characteristics, both of which are related to the modal properties of the structure. The techniques presented in this paper are important to golf club manufacturers attempting to produce clubs with improved performance within shorter NPI timescales and will ultimately provide additional performance predicting capability at an early stage in the design process. In particular, this paper demonstrates :

i) The level of geometric modelling and finite element discretisation accuracy required to create a finite element model of a hollow golf club head suitable for performance prediction, with references to other related publications in which the details of the modelling procedure are documented.

ii) The detailed experimental hollow golf club head modal and impact data obtainable by application of non-contacting laser Doppler vibrometry and electronic speckle pattern interferometry techniques and their application to finite element model validation. The use of these experimental techniques in the golf industry has not been reported previously.

iii) The different modal characteristics of the three surfaces which comprise the majority of the hollow structure and their influence on the impact sound.

iv) The potential for quantification of further components of the club-ball impact with respect to the 'feel' of the golf shot and for correlation of mechanical behaviour with functional performance. 


\section{Acknowledgements}

The authors would like to acknowledge the support of the Engineering and Physical

Sciences Research Council, Dunlop Slazenger International Limited and Delcam International plc. The contribution to club head modelling made by David Underwood of the Manufacturing Engineering Department at Loughborough University is also appreciated.

\section{References}

1. Braham,J (1992): Super clubs hit the sweet spot. Machine Design, April 1992. 3034.

2. Chou,PC; Liang,D; Yang,J (1994): Contact forces, coefficient of restitution and spin rate of a golf ball impact. Science and Golf 2, Proceedings of the World Scientific Congress of Golf. St Andrews, UK. (Eds: Cochran,AJ; Farrally,MR). 296-301.

3. Iwata,M; Okuto,N; Satoh,F (1990): Designing of golf club heads by finite element method (FEM) analysis. Science and Golf, Proceedings of the First World Scientific Congress of Golf. St Andrews, UK. (Ed: Cochran,AJ). 274-279.

4. Stead,A (1992): Analysis of the impact stresses on a metal golf wood using finite element methods. BEng project report, Dept. of Manufacturing Engineering, Loughborough University, UK.

5. Thomson,RD; Whittaker,AR; Wong,K; Adam,A (1990): Impact of a golf ball with a rigid clubface. Proceedings of the 6th UK ABAQUS User Group Conference; September 27, 1990; University of Manchester, UK. (Eds: Brundrett,S; Redhead,JG). 41-47.

6. Mather,JSB (1996): The role of club response in the design of current golf clubs. Proceedings of the 14th International Modal Analysis Conference, Dearborn, Michigan, USA. Vol. 1. 397-403.

7. Okubo,N; Simada,M (1990): Application of CAE (Computer Aided Engineering) to golf club dynamics. Science and Golf, Proceedings of the First World Scientific Congress of Golf. St Andrews, UK (Ed: Cochran,AJ), UK. 270-273.

8. Swider,P; Ferraris,G; Vincent,B (1994): Theoretical and experimental dynamic behaviour of a golf club made of composite material. Modal Analysis : International Journal of Analytical and Experimental Modal Analysis 9, 57-69. 
9. Thomas,G; Deiters,T; Best,C (1995): Simulating Golf Club Performance using Modal Analysis. Proceedings of the 13th International Modal Analysis Conference. Vol. 2. 989-995.

10. Varoto,PS; McConnell,KG (1995): Using modal analysis to evaluate golf club performance. Sound and Vibration, March 1995, 20-23.

11. Hocknell,A; Jones,R; Rothberg,SJ (1996): Experimental analysis of impacts with large elastic deformation. Part 1 : Linear motion. Measurement Science and Technology. Vol.7 No.9. 1247-1254.

12. Hocknell,A; Jones,R; Rothberg,SJ (1996): Engineering 'feel' in the design of golf clubs. The Engineering of Sport : Proceedings of the 1st International Conference on the Engineering of Sport, Sheffield, UK. (Ed: Haake,SJ). 333-337.

13. Mitchell,SR; Newman,ST; Hinde,CJ; Jones,R (1994): A design system for iron golf clubs. Science and Golf 2, Proceedings of the World Scientific Congress of Golf. St Andrews, UK. (Eds: Cochran,AJ; Farrally,MR). 390-395.

14. Mitchell,SR (1996): A feature based approach to the computer aided design of sculptured products. Ph.D. Thesis, Loughborough University, UK.

15. Hocknell,A; Mitchell,SR; Underwood,DJ; Jones,R (1996): Feature based quadrilateral mesh generation for sculptured surface products. Submitted to the Journal of Design and Manufacturing.

16. Ewins,DJ (1984): Modal Testing Theory and Practice. Research Studies Press, England.

17. Jones,R; Wykes,C (1983): Holographic and Speckle Interferometry. Cambridge University Press. 


\section{List of figure captions}

Figure 1 - Hollow golf club head feature anatomy

Figure 2 - Golf club head wireframe model

Figure 3 - Discretised finite element golf club head model

Figure 4 - Frequency response functions for points on the face, crown and sole features

Figure 5a - First mode of the face observed using ESPI $(5.64 \mathrm{kHz})$

Figure $5 \mathrm{~b}$ - First mode of the face calculated by the finite element method $(5.51 \mathrm{kHz})$

Figure 6 - Example ESPI and calculated crown mode $(5.96 \mathrm{kHz})$

Figure 7 - Example ESPI and calculated sole mode $(8.20 \mathrm{kHz})$

Figure 8 - Experimentally measured club head acceleration during impact and finite element model equivalent result

Figure 9 - Measured sound spectrum for a central club-ball impact

Figure 10 - Antinode position variation on face modeshapes

Figure 11 - Measured sound spectrum for an off-centre club-ball impact 


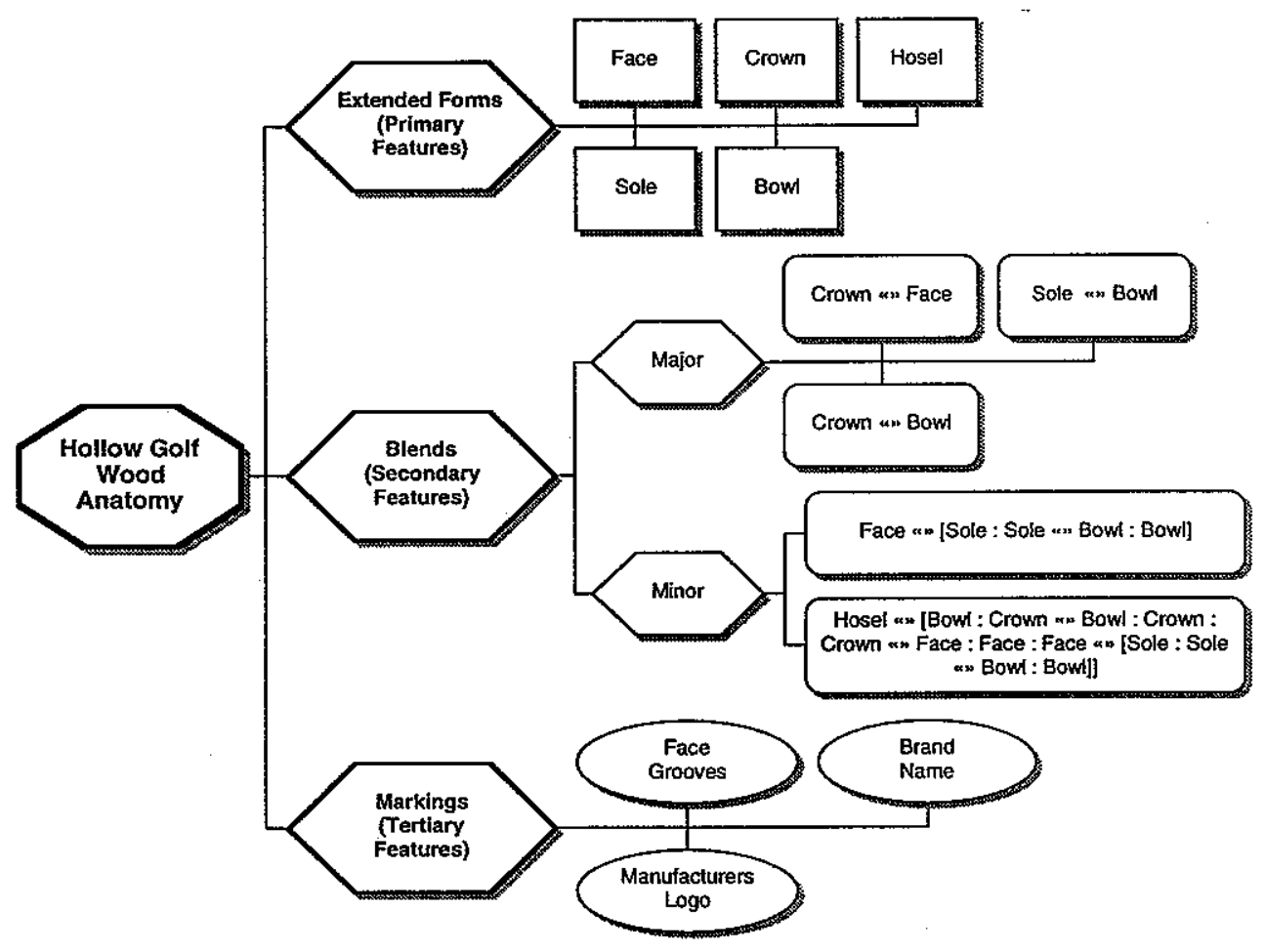

Figure 1

Hocknell, Mitchell, Jones and Rothberg Loughborough University, UK 


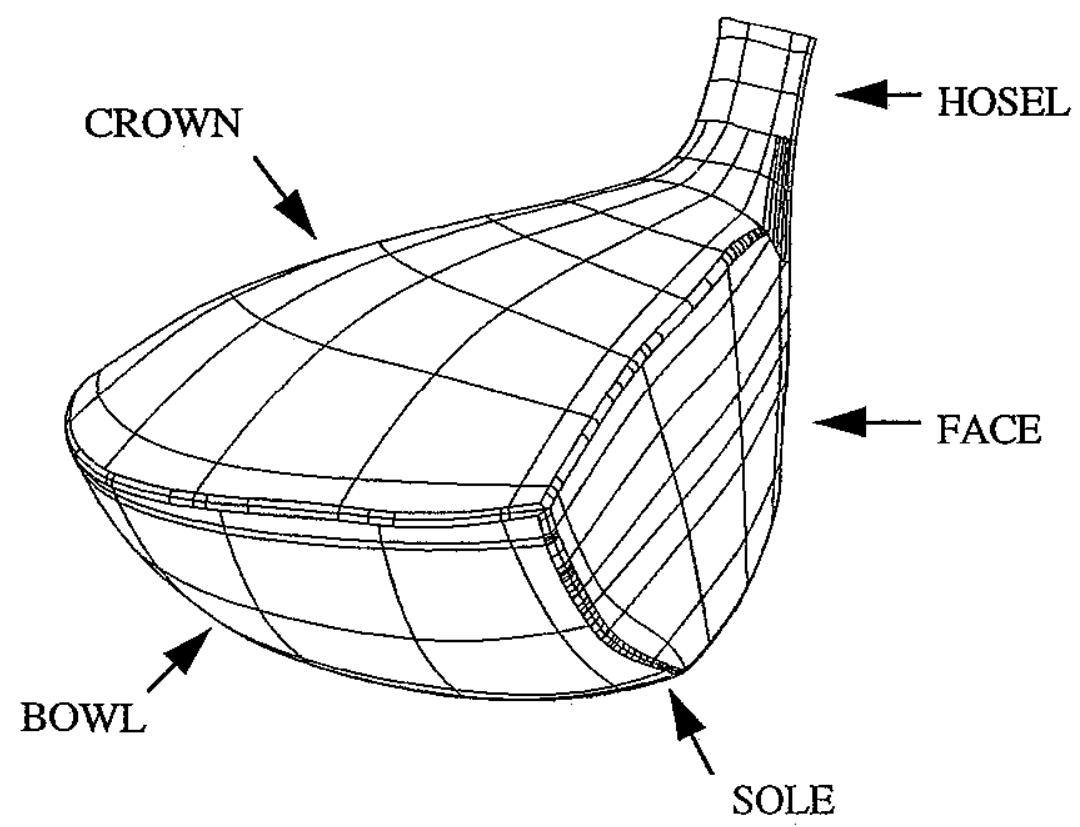

i.

Figure 2

Hocknell, Mitchell, Jones and Rothberg

Loughborough University, UK 


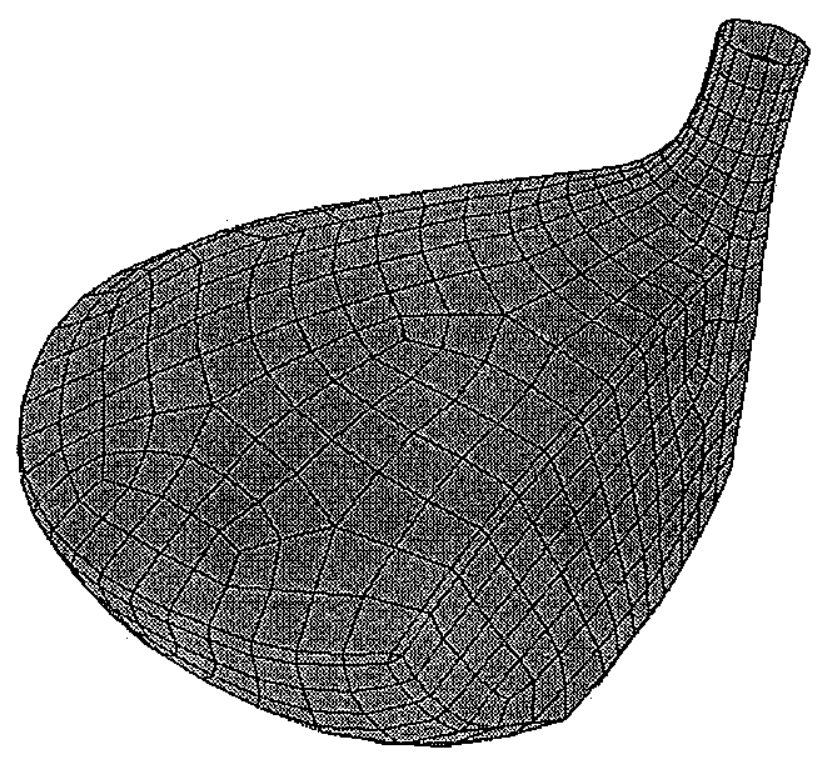

Figure 3

Hocknell, Mitchell, Jones and Rothberg

Loughborough University, UK 


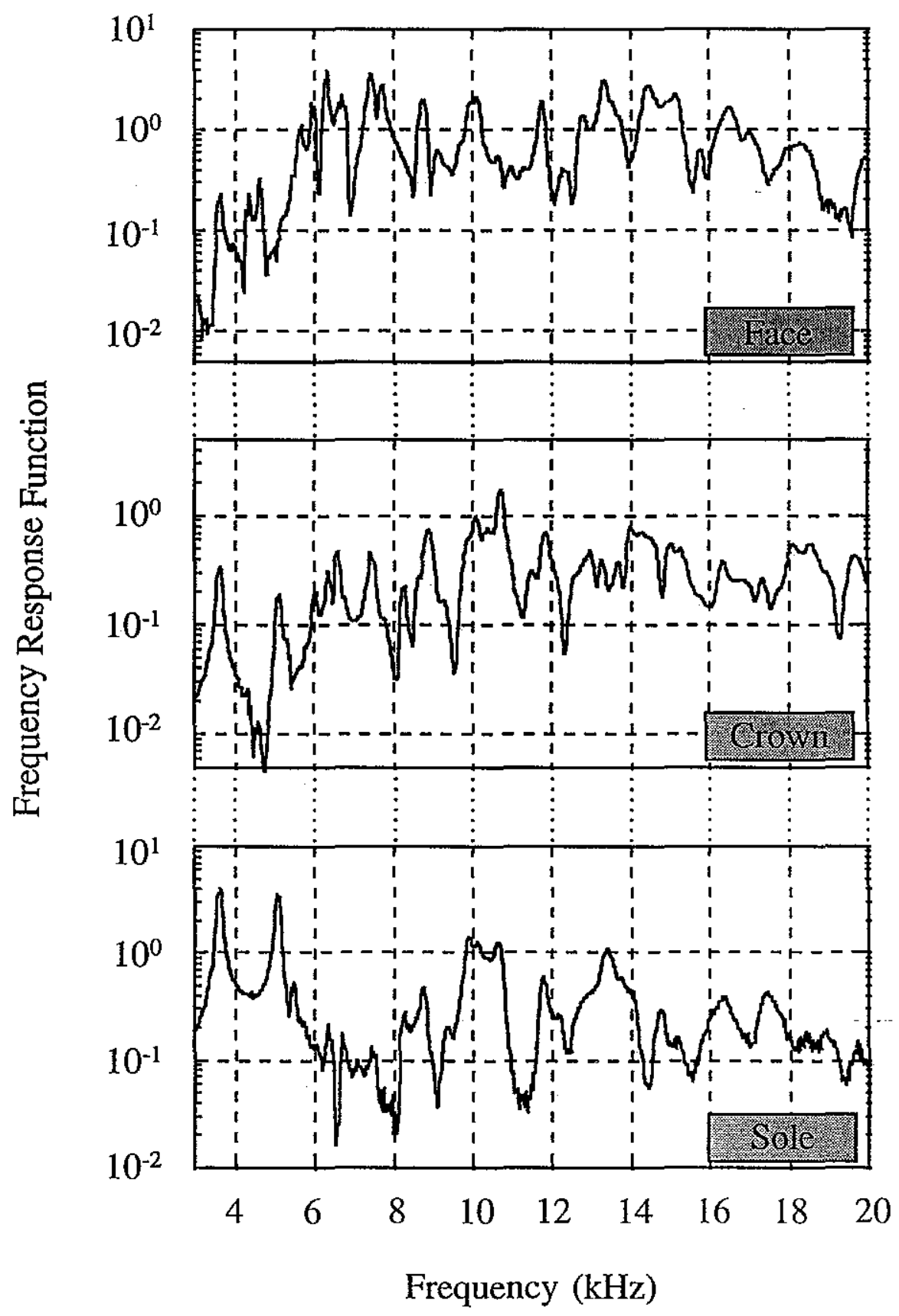

Figure 4

Hocknell, Mitchell, Jones and Rothberg Loughborough University, UK 


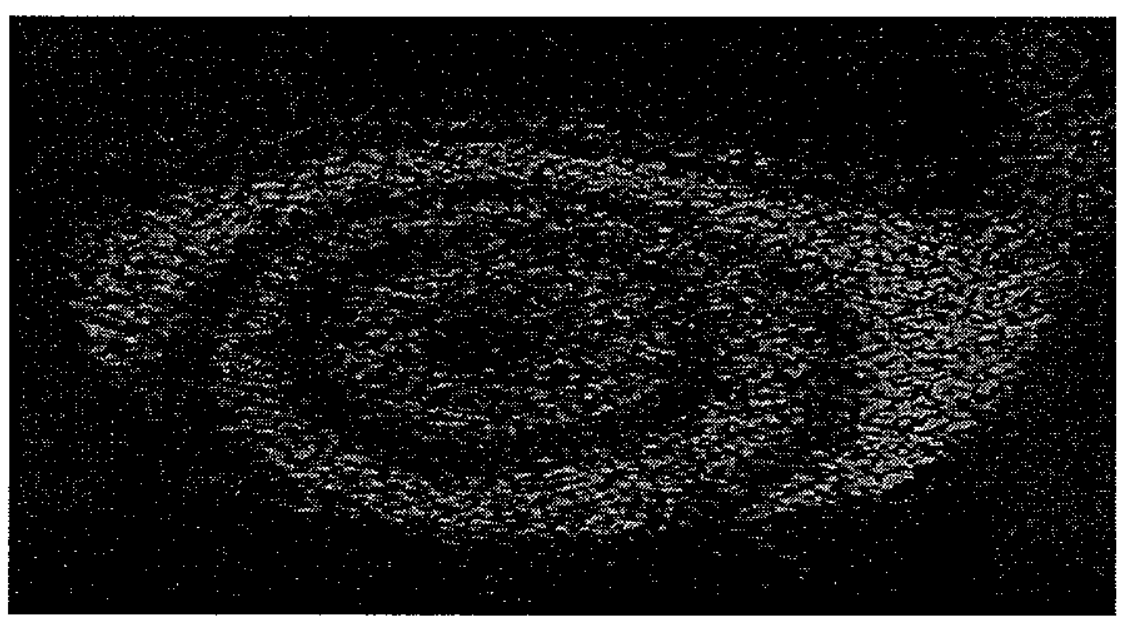

Figure 5a

Hocknell, Mitchell, Jones and Rothberg

Loughborough University, UK 


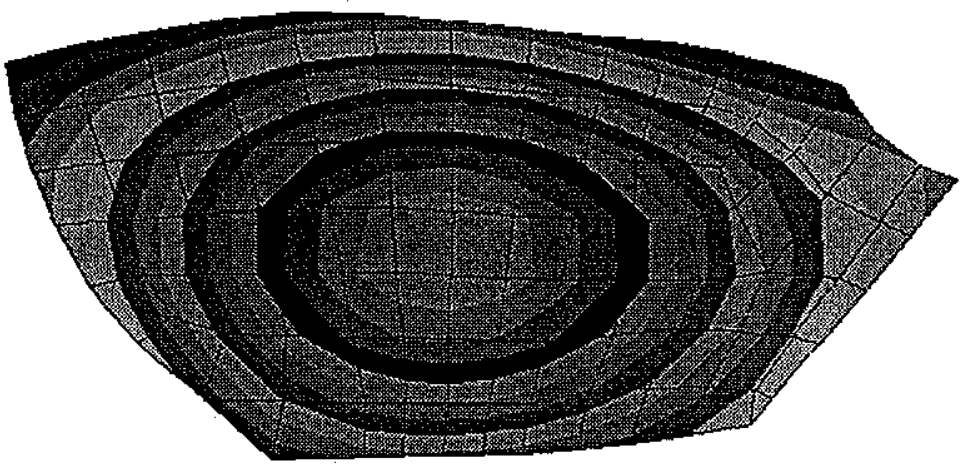

Figure $5 b$

Hocknell, Mitchell, Jones and Rothberg

Loughborough University, UK 


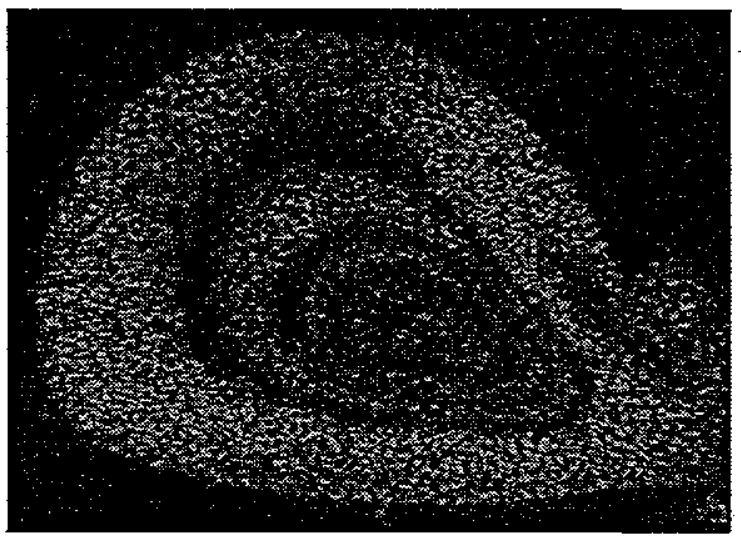

ßin

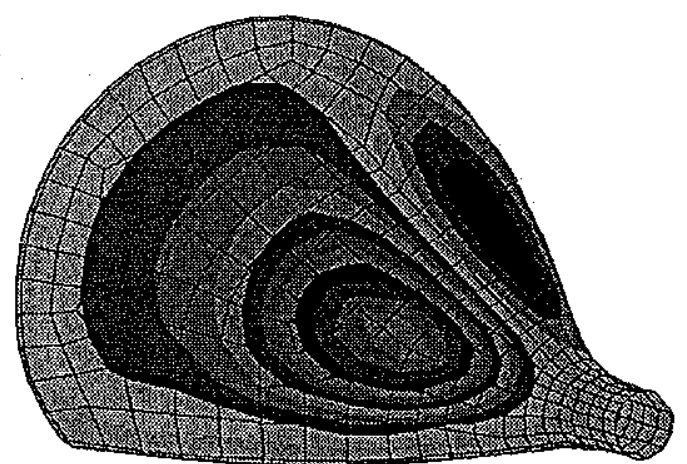

Figure 6

Hocknell, Mitchell, Jones and Rothberg

Loughborough University, UK 

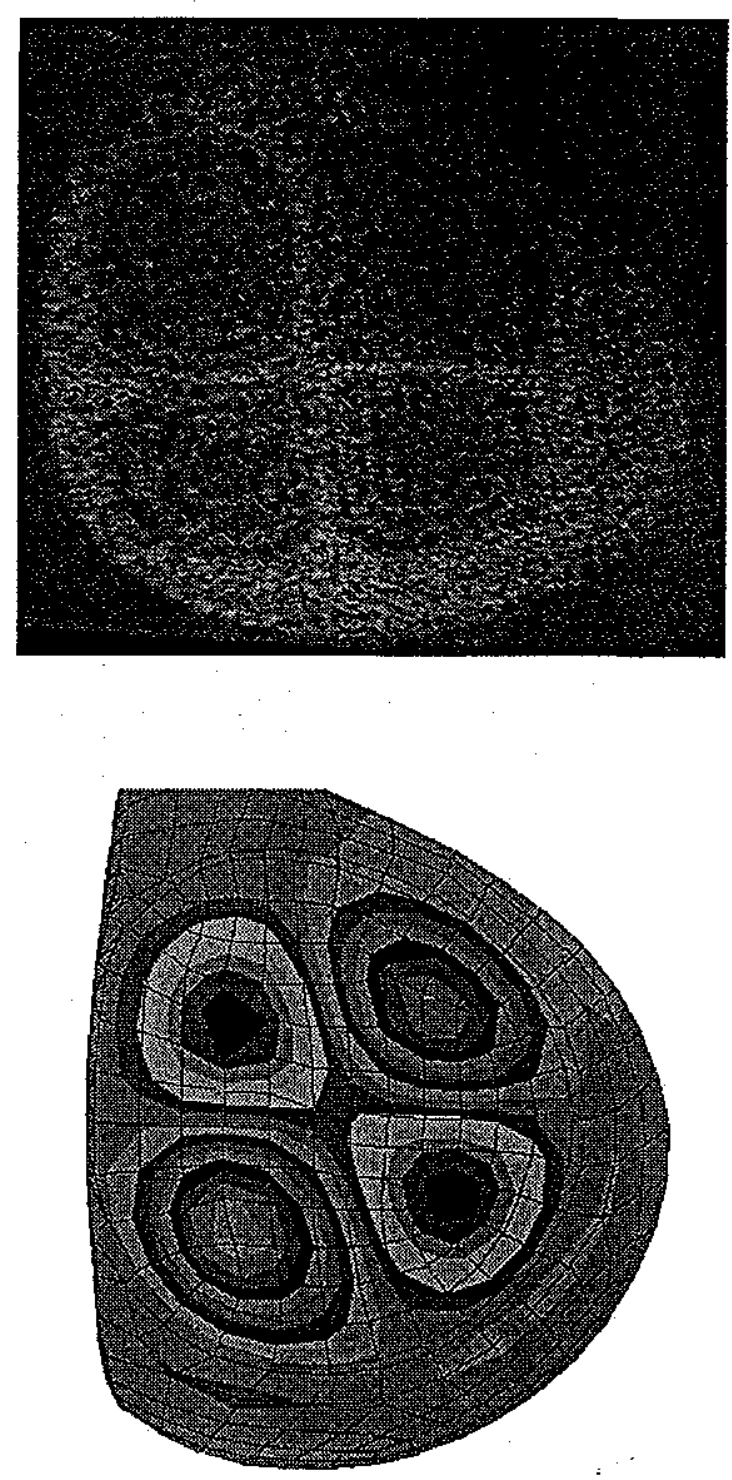

Figure 7

Hocknell, Mitchell, Jones and Rothberg Loughborough University, UK 


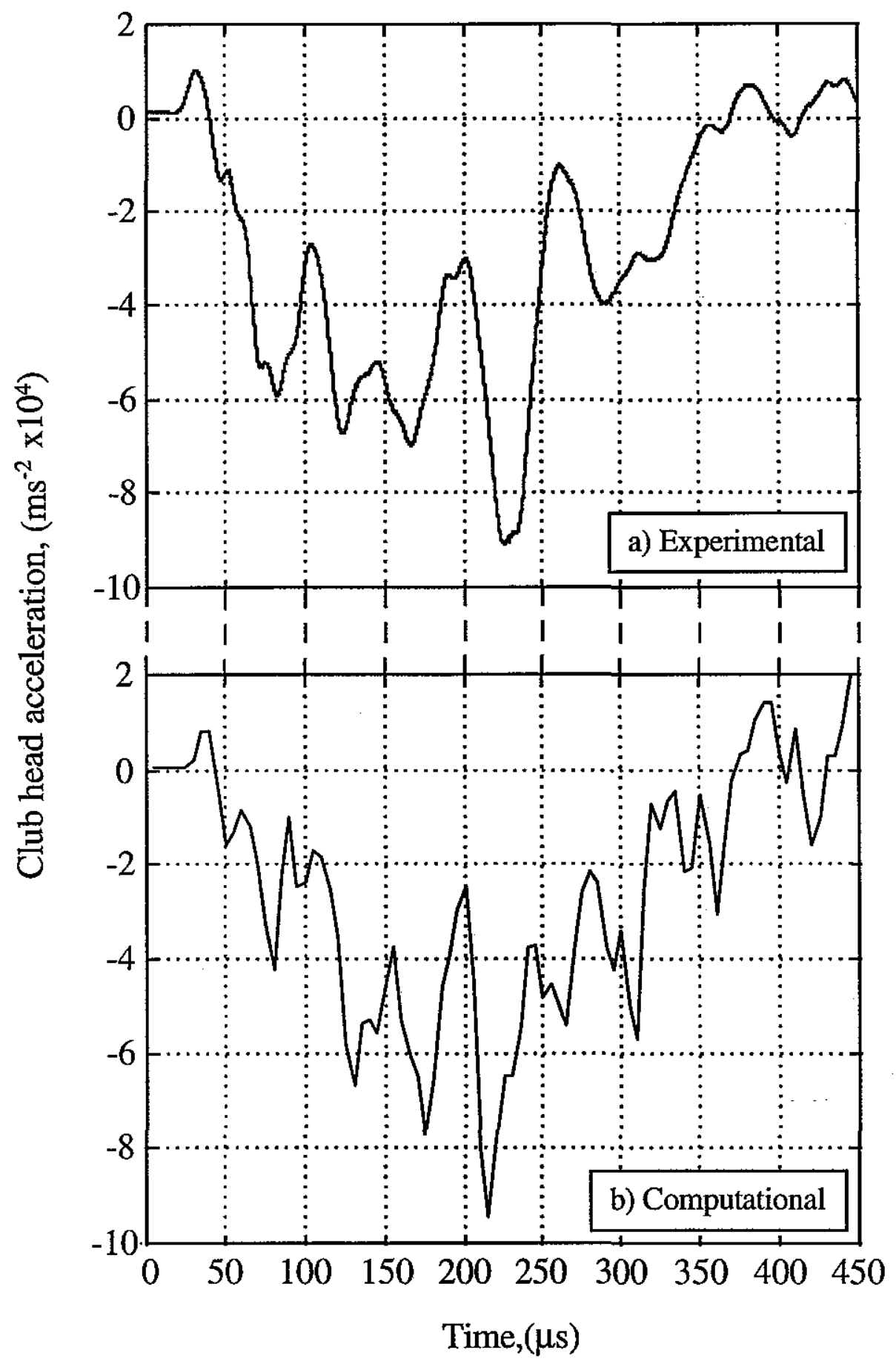

Figure 8

Hocknell, Mitchell, Jones and Rothberg Loughborough University 


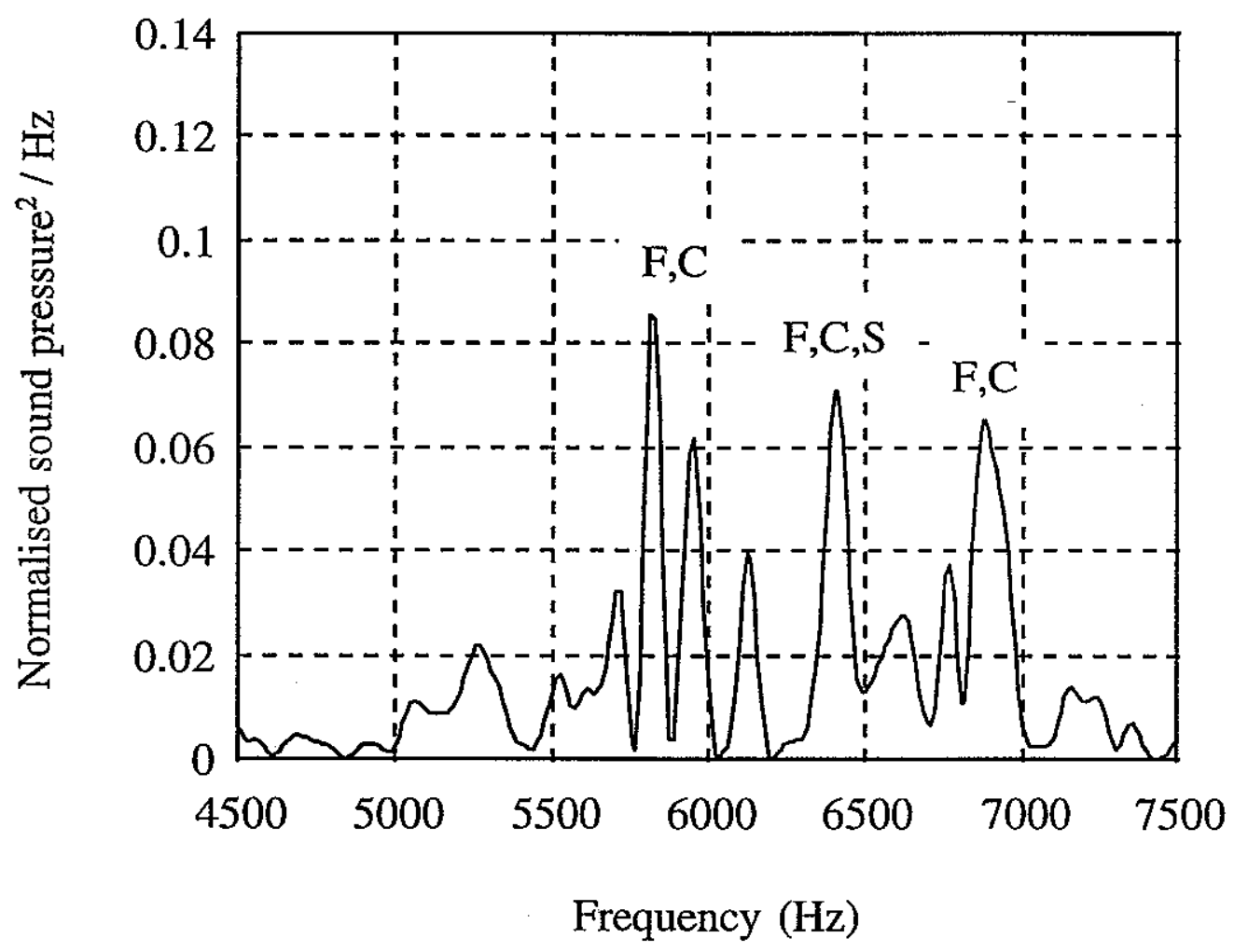

Figure 9

Hocknell, Mitchell, Jones and Rothberg Loughborough University, UK 


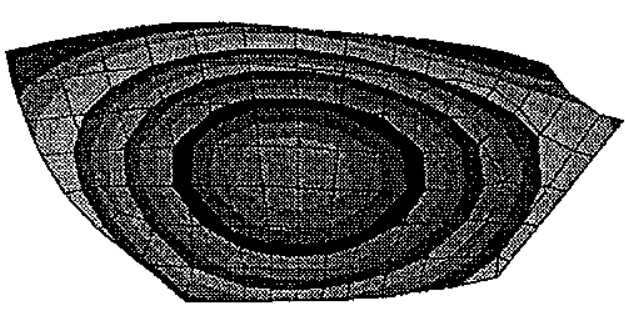

$5.9 \mathrm{kHz}$ face mode

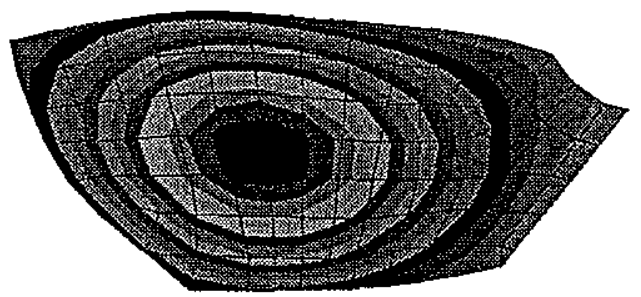

$6.4 \mathrm{kHz}$ face mode

Figure 10

Hocknell, Mitchell, Jones and Rothberg Loughborough University, UK 


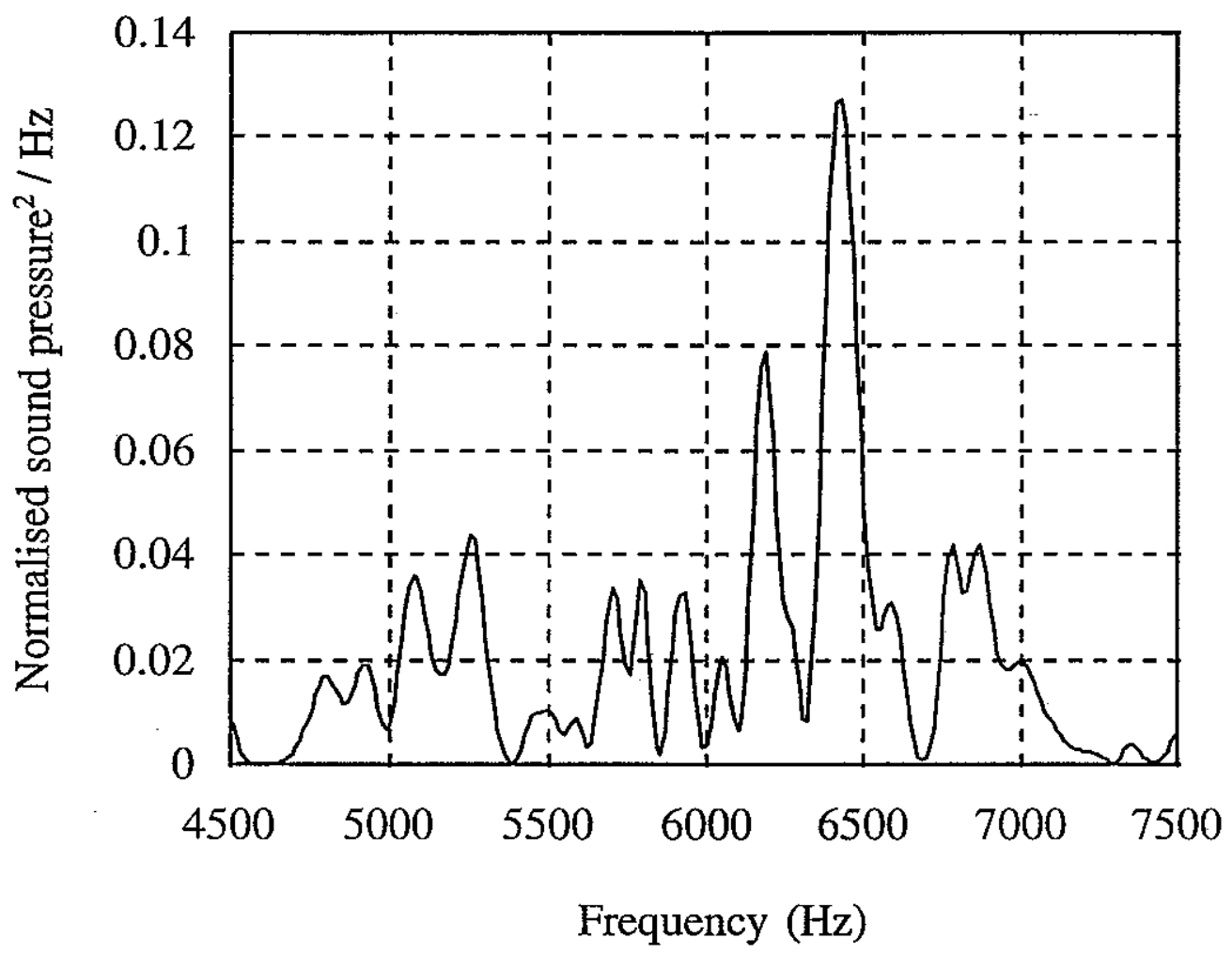

Figure 11

Hocknell, Mitchell, Jones and Rothberg

Loughborough University, UK 